\title{
A Short-Term Measure of Dark Energy in Sparse Atomic Estimations
}

\author{
Bob L. Sturm and John J. Shynk \\ Department of Electrical and Computer Engineering \\ University of California \\ Santa Barbara, CA 93106-9560
}

\author{
Laurent Daudet \\ Laboratoire d'Acoustique Musicale \\ Université Pierre et Marie Curie - Paris 6 \\ 75015 Paris, France
}

\begin{abstract}
A sparse atomic estimation involves finding a representation of a signal that uses terms with finite support drawn from an overcomplete dictionary. These terms can constructively and destructively interfere with each other in the reconstruction, and some may even disappear. For this reason, we refer to the interference between atoms in the sparse estimation as "dark energy" (DE). Building upon our previous work, we present a short-term measure of DE (STDE) that can be used to examine the distribution of interference in the signal estimation. We present results for several signals, and discuss some applications of this measure.
\end{abstract}

\section{INTRODUCTION}

A sparse atomic estimation approximates a signal to a specified precision by a linear combination of terms with finite support, called atoms, selected from an overcomplete set, called a dictionary. Sparse decomposition methods include $L_{1}$ minimization, such as basis pursuit [1], and greedy iterative descent strategies, such as matching pursuit (MP) [2]. The motivations for developing sparse estimations include sparsity, efficiency, robustness, and meaningfulness. They have found use in several areas, such as coding [3], source separation [4], and visualization and transformation of audio signals [5].

Decomposing signals with an overcomplete dictionary using MP often produces terms that "correct" previous terms through destructive interference [6]-[9]. Thus, although an atom exists in an estimation, its superposition in the signal reconstruction might result in its partial disappearance, thus contributing little energy to, or even removing energy from, the approximation. Because of this effect, we refer to the constructive and destructive interference between terms of an estimation as dark energy (DE) [9]. We are interested in using this phenomenon to interpret the signal, examine its coherence with the dictionary, and evaluate the performance of the decomposition algorithm.

Previously, we formally defined DE, investigated some of its properties, and studied its behavior for various audio signals [9]. In this paper, after reviewing sparse atomic estimation and $\mathrm{DE}$, we build upon these results to create a measure of the interference within an estimation that is localized with respect to the signal itself, ultimately denoting where and to what degree an estimation corrects itself. This short-term dark energy (STDE) measure could be applied to increase the efficiency of an estimation and the performance of a decomposition algorithm by discriminating between portions of the signal that do and do not "make sense" with respect to the dictionary i.e., whether or not signal features are contained in the dictionary.

\section{Sparse Atomic Estimation}

The signal vector $\mathrm{x}$ of length $M$ can be estimated by a linear combination of vectors (atoms) selected from the columns of a dictionary $\mathbf{D}$ consisting of $N$ unit-norm vectors of length $M$ defined a priori, where $N \gg M$ usually. The $n$ th-order representation of $\mathrm{x}$ is given by

$$
\mathbf{x}=\mathbf{G}(n) \mathbf{c}(n)+\mathbf{r}(n)=\widetilde{\mathbf{x}}(n)+\mathbf{r}(n)
$$

where $\mathbf{G}(n)=\left[\mathbf{g}_{0}|\cdots| \mathbf{g}_{n-1}\right]$ consists of $n$ columns selected from the dictionary, and $\mathbf{c}(n)=\left[c_{0}, \ldots, c_{n-1}\right]^{T}$ is a column vector of the expansion coefficients. The $n$ th-order estimation is $\{\mathbf{G}(n), \mathbf{c}(n)\}$, which is evaluated to produce the $n$ th-order approximation $\widetilde{\mathbf{x}}(n)=\mathbf{G}(n) \mathbf{c}(n)$. The $n$ th-order residual $\mathbf{r}(n)=\mathbf{x}-\widetilde{\mathbf{x}}(n)$ is that part of $\mathbf{x}$ yet to be represented by the estimation (with $\mathbf{r}(0) \equiv \mathbf{x}$ ). Note that $n$ refers to the order of the estimation process, or the decomposition iteration, and is not a time index.

MP is a simple iterative descent algorithm that solves (1) [2]. It estimates $\mathrm{x}$ by concatenating $\mathrm{G}(n)$ with atoms selected from $\mathbf{D}$ based on the following criterion:

$$
\mathbf{g}_{n}=\arg \max _{\mathbf{g}=\mathrm{De}_{j}}\left|\mathbf{g}^{H} \mathbf{r}(n)\right|, \quad j=1,2, \ldots, N
$$

where $\mathrm{e}_{j}$ is the $j$ th unit vector (with 1 in the $j$ th row), and the superscript $H$ denotes complex conjugate transpose. The matrix of selected atoms is updated as $\mathbf{G}(n+1)=\left[\mathbf{G}(n) \mid \mathbf{g}_{n}\right]$, and the new expansion coefficient is appended to the end of $\mathrm{c}(n)$. The new atom is weighted and subtracted from the current residual, and the new residual $\mathbf{r}(n+1)$ is used to find the next atom via (2). To minimize the energy of the residual at each iteration, the expansion coefficient is computed as

$$
c_{n}=\mathbf{g}_{n}^{H} \mathbf{r}(n) \text {. }
$$

MP continues to build the estimation until convergence, or when another criterion is met, such as a minimum signal-toresidual ratio. MP is called "greedy" because it maximizes the amount of energy it removes from the residual without regard to past or future iterations, i.e., it is a local optimization.

In this paper, we use a dictionary of real Gabor atoms. A Gabor atom is a truncated Gaussian window that is scaled, translated, modulated, and discretized as follows:

$$
g(k ; u, s, f, \phi)=A w(k-u ; s) \cos (2 \pi f(k-u) T+\phi),
$$




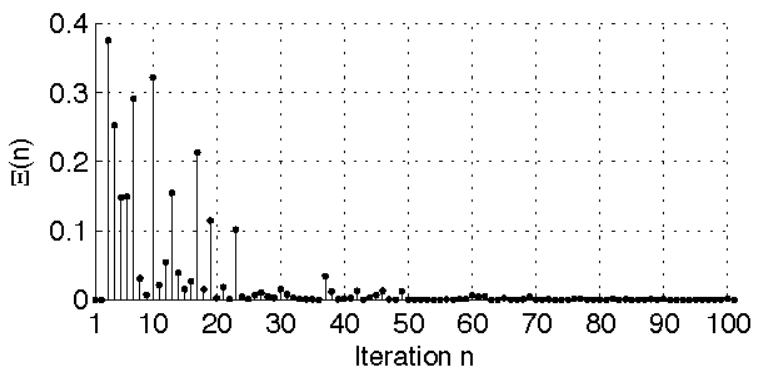

Fig. 1. An example of $\mathrm{DE}$ as a function of iteration (estimation order).

for the time index $0 \leq k \leq M-1$, where $T$ is the sampling period, $0 \leq u<M-s / 2$ is the translation in samples, $1 \leq s \leq M$ is the scale in samples, and $0 \leq f \leq 0.5$ and $0 \leq \phi \leq 2 \pi$ are the (normalized) modulation frequency and phase, respectively. The scalar normalization factor $A$ is assigned such that $\sum_{k=0}^{M-1}|g(k ; u, s, f, \phi)|^{2}=1$. The function $w(k ; s)$ is a translated and discretized Gaussian window truncated to $s$ samples:

$$
w(k ; s)= \begin{cases}\exp \left(-\frac{(k-s / 2)^{2}}{2(\alpha s)^{2}}\right), & k=0,1, \ldots, s-1 \\ 0, & \text { else }\end{cases}
$$

where the constant $\alpha$ controls the variance of the window. The $(k+1)$ st element of $\mathbf{g}$ is $g(k ; u, s, f, \phi)$.

\section{INTERFERENCE AND DARK ENERGY}

A pair of vectors $\left\{\mathbf{g}_{i}, \mathbf{g}_{j}\right\}$ "interfere" with each other if

$$
\left\|\mathbf{g}_{i}+\mathbf{g}_{j}\right\|^{2} \neq\left\|\mathbf{g}_{i}\right\|^{2}+\left\|\mathbf{g}_{j}\right\|^{2}
$$

i.e., constructive and destructive interference between two vectors results in more or less energy, respectively, than that of their sum. Clearly, any pair of non-orthogonal vectors have this interference property. We propose that the interference within a sparse atomic estimation be measured by its DE, which we have defined specifically for MP and the signal model in (1) [9]. At the $n$th iteration, the $\mathrm{DE}$ associated with the new atom $\mathbf{g}_{n-1}$ is the magnitude of the difference between the energy of the new approximation, $\|\widetilde{\mathbf{x}}(n)\|^{2}=\left\|\widetilde{\mathbf{x}}(n-1)+c_{n-1} \mathbf{g}_{n-1}\right\|^{2}$, and the energy of the approximation that would result if the new atom was orthogonal to the current approximation, $\|\widetilde{\mathbf{x}}(n-1)\|^{2}+\left|c_{n-1}\right|^{2}$. Thus, the DE associated with $\mathbf{g}_{n-1}$ at the $n$th iteration is

$$
\Xi(n)=||\left|\widetilde{\mathbf{x}}(n) \|^{2}-\left(\|\left.\widetilde{\mathbf{x}}(n-1)\right|^{2}+\left|c_{n-1}\right|^{2}\right)\right|,
$$

for $n \geq 1$, which is equivalent to the magnitude of the difference of the two sides in (6). Rearranging terms, (7) can be simplified to [9]

$$
\Xi(n)=2\left|c_{n-1} \mathbf{g}_{n-1}^{H} \widetilde{\mathbf{x}}(n-1)\right|,
$$

from which we see that the DE associated with a new atom is proportional to the amount it already exists in the current approximation.

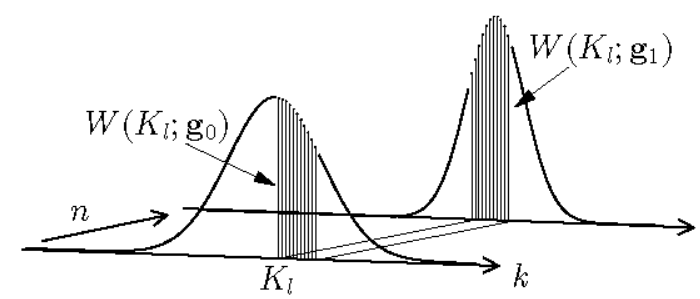

Fig. 2. The contributions of DE by the atoms to segment $K_{l}$ are weighted by the fraction of energy in the corresponding envelopes. The arrows denote the samples of the envelopes that are used in (11).

\section{SHORT-TERM DARK ENERGY}

The measure in (8) indicates where interference occurs in the decomposition process, as seen in Fig. 1. However, we want to know where it is occurring with respect to the signal itself. Such a measure can help discriminate between portions of a signal that "make sense" with respect to the dictionary, e.g., display less interference, and portions that are difficult to decompose using the dictionary. Ultimately we want to use this information to adapt the decomposition algorithm to the signal, and to optimize its estimation.

Consider partitioning the discrete-time region $\mathcal{K}$ of $\mathrm{x}$ into $L$ segments, i.e., $\mathcal{K}=\bigcup_{l=1}^{L} K_{l}$ where $K_{l}=\left[k_{l}, k_{l+1}\right)$ and $k_{l}<k_{l+1}$ are the sample indices. We calculate the total DE of each segment as a weighted sum of the DE associated with each atom of the estimation. Collecting these totals together yields the short-term dark energy (STDE). Formally, for an $n$ th-order estimation, the STDE is given by

$$
\Xi_{S T}(k ; n)=\sum_{l=1}^{L} I_{\left[k_{l}, k_{l+1}\right)}(k)\left[\sum_{i=1}^{n} W\left(K_{l} ; \mathbf{g}_{i-1}\right) \Xi(i)\right]
$$

where $W\left(K_{l} ; \mathbf{g}_{i}\right)$ is a weight in $K_{l}$ associated with $\mathbf{g}_{i}$, and $I_{\left[k_{l}, k_{l+1}\right)}(k)$ is the indicator function.

There are many possible weightings. Denote the support of atom $\mathbf{g}_{i}$ as $G_{i} \subseteq \mathcal{K}$. For example, we might use

$$
W\left(K_{l} ; \mathbf{g}_{i}\right)=\frac{\mathcal{L}\left\{G_{i} \cap K_{l}\right\}}{\mathcal{L}\left\{G_{i}\right\}} \leq 1
$$

where $\mathcal{L}\left\{\left[k_{l}, k_{l+1}\right)\right\}=k_{l+1}-k_{l}$ is the Lebesgue measure. In effect, this weighting assumes that the DE contributed by an atom is uniformly distributed over its support. For a Gabor atom, this probably is not appropriate because the degree of interference near its tail should be less than that near its mean. Instead, we make the assumption that DE is spread over an atom in proportion to the energy of its envelope. For a Gabor atom $\mathbf{g}_{i}$, this weighting is determined by the fraction of energy of $w\left(k ; s_{i}\right)$ in $(5)$ over $K_{l}$ :

$$
W\left(K_{l} ; \mathbf{g}_{i}\right)=\frac{\sum_{k \in K_{l}}\left|w\left(k-u_{i} ; s_{i}\right)\right|^{2}}{\sum_{k \in \mathcal{K}}\left|w\left(k ; s_{i}\right)\right|^{2}} .
$$

Figure 2 shows an example of this weighting for $n=2$. 


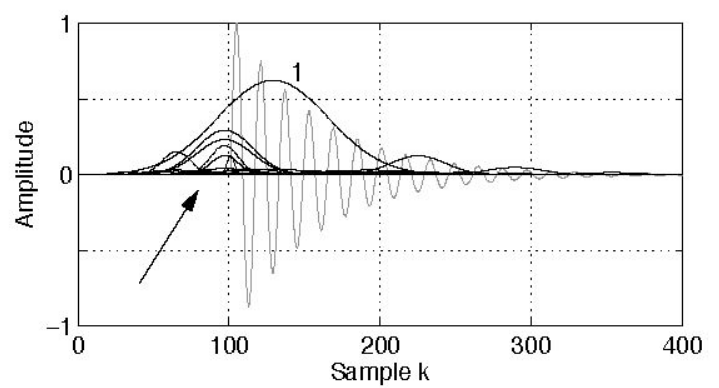

(a) Interference preceding an attack.

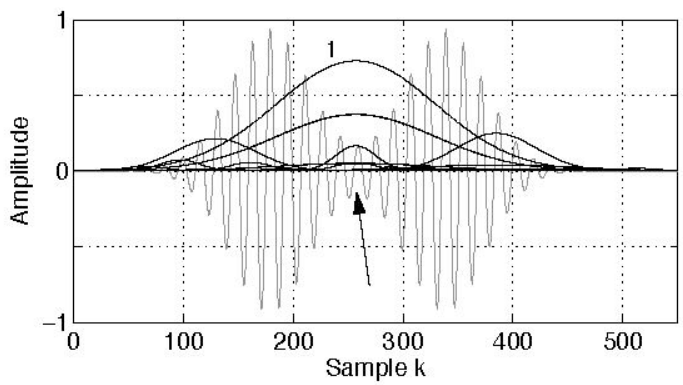

(b) Interference between modes.

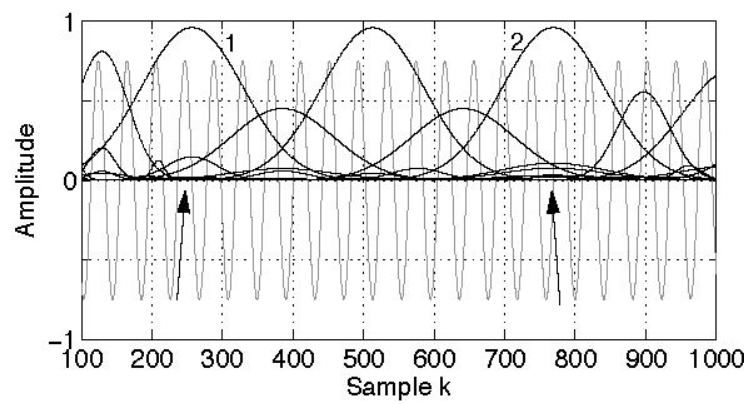

(c) Interference during steady state.

Fig. 3. Each of these waveforms (gray) is decomposed by MP into several Gabor atoms (the envelopes are shown in black). Arrows point to regions of destructive interference.

\section{EMPIRICAL RESULTS}

Figure 3 shows three simple examples of how interfering terms arise in a sparse atomic decomposition using MP. The asymmetric signal in Fig. 3(a) is a decaying exponential and has a sharp attack. The first atom selected by MP (labeled 1) partially extends into the zero-energy region preceding the attack. To remove the energy introduced in that region, MP selects several terms in later iterations (denoted by the arrow) to destructively interfere with and remove that portion of the initial atom. Figure 3(b) shows the decomposition of an amplitude-modulated (AM) signal. The first atom selected by MP inadvertently links the two modes together, which results in terms that destructively interfere to preserve the original waveform. The decomposition of a sinusoidal signal also exhibits interference. Figure 3(c) shows how the first two atoms overshoot the waveform envelope, and subsequent atoms destructively interfere to reduce the corresponding error.

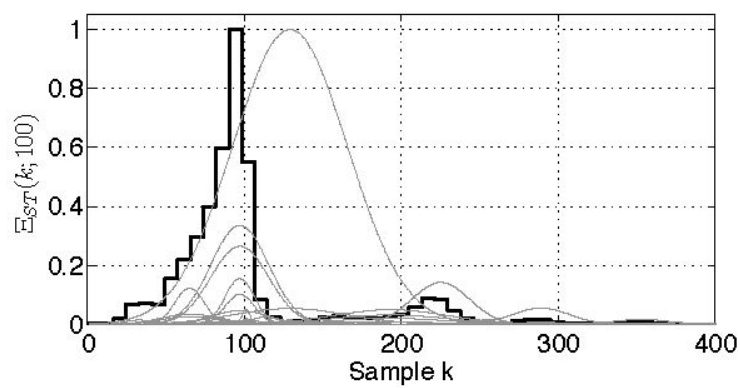

(a) Interference preceding an attack.

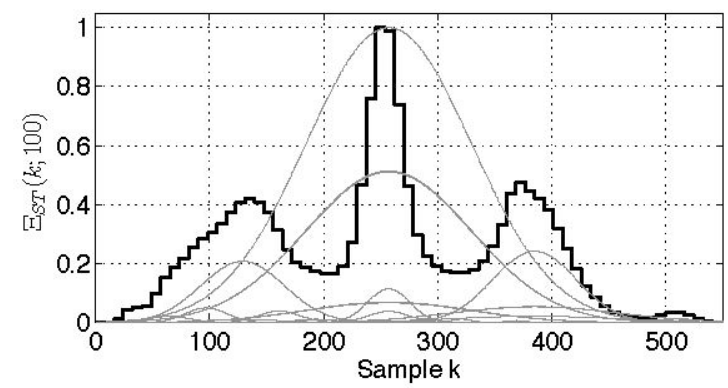

(b) Interference between modes.

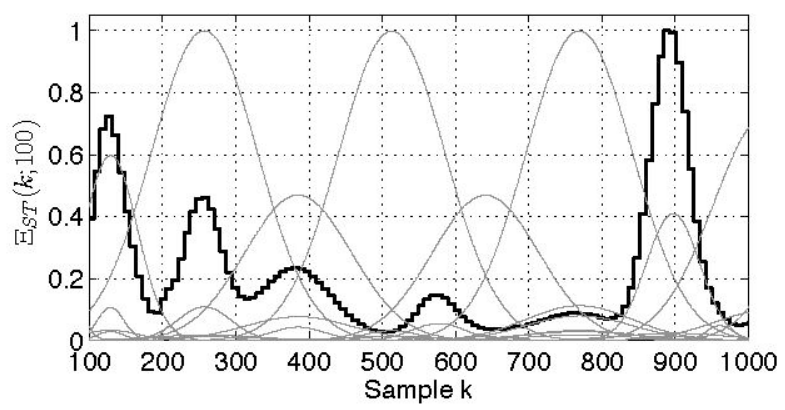

(c) Interference during steady state.

Fig. 4. The normalized STDE is shown (in black) for the estimation of each waveform in Fig. 3. The envelopes of the atoms are scaled relative to the maximum normalized STDE and are overlaid (using gray lines).

Figure 4 shows the STDE for each of these signals using a partition length of eight samples, i.e., $\mathcal{L}\left\{K_{l}\right\}=8$. For the signal with an attack, Fig. 4(a) shows that DE is concentrated around the attack. Similarly, the concentration of DE between the two modes of the AM signal is clearly seen. The DE prior to and after the modes is due to atoms constructively interfering with the tapered sides of the initial atom. For the sinusoid, the DE exhibits a much more complex behavior. Small increases in the DE occur near the centers of the first two atoms selected, but some of the larger values appear to be caused by constructive interference, e.g., the atoms centered around sample $k=900$.

Using the same Gabor dictionary, we decomposed a music signal and found its STDE. The top part of Fig. 5 shows the wivigram for the signal of a glockenspiel (struck metal bars) playing a short melody. A wivigram [2], [5] is a superposition of the Wigner-Ville distribution of each weighted time- 


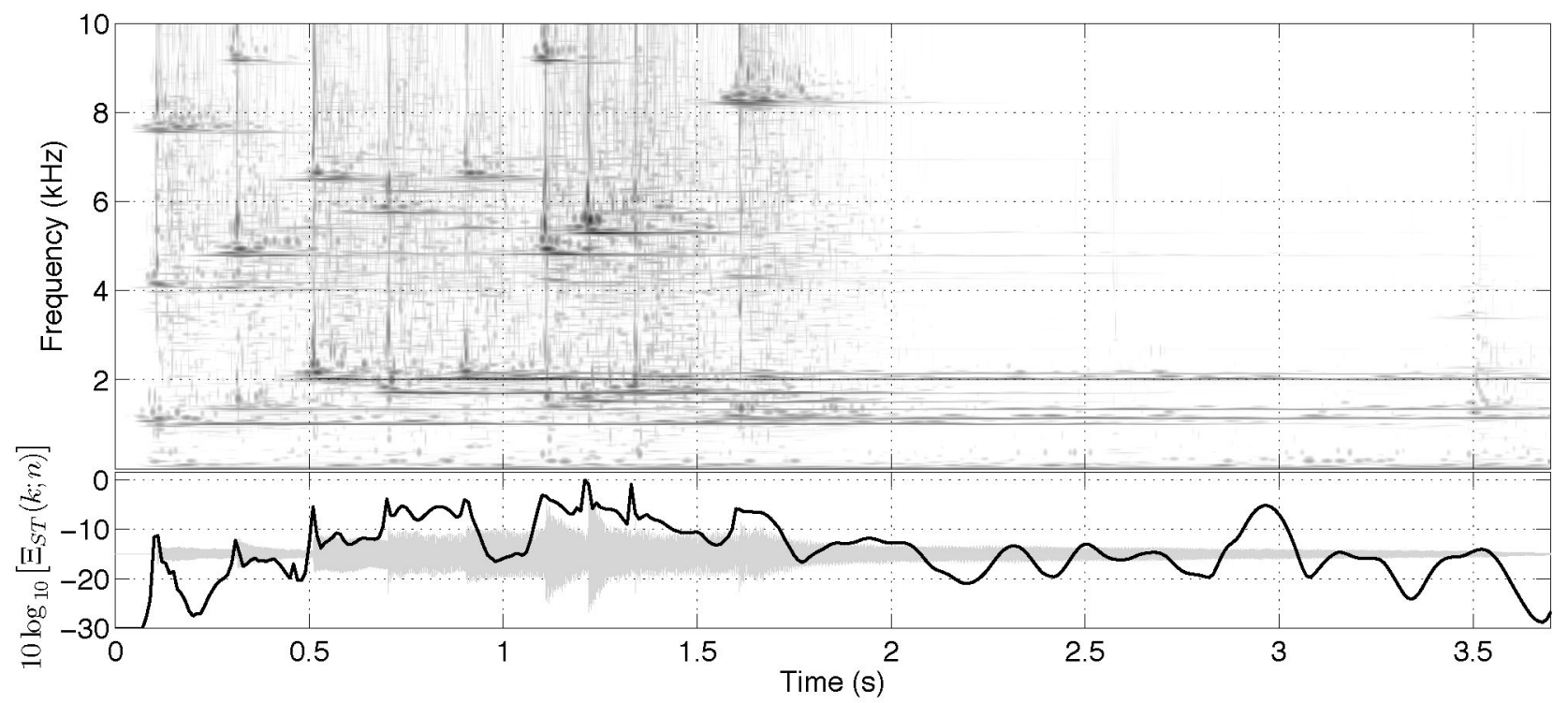

Fig. 5. An MP decomposition using a Gabor dictionary of a music signal is visualized using a wivigram (top). The log-normalized STDE of the estimation is shown as the dark line overlaid on the time-domain waveform (bottom). The order of the estimation is $n=41,293$.

frequency atom in an estimation $\{\mathbf{G}(n), \mathbf{c}(n)\}$. This signal is characterized by sharp attacks and ringing harmonic tones. The multiresolution representation created by MP contains wideband small-scale atoms near the attacks, and large-scale atoms for the ringing parts. The STDE, calculated using a partition length of $10 \mathrm{~ms}$ (441 samples), is shown superimposed on the audio waveform at the bottom of the figure. As was seen for the simple signal in Fig. 3(a), sharp changes in DE occur near the attacks. In regions that do not have attacks, such as that beyond $2 \mathrm{~s}$, the change in DE is much more smooth. The high concentration of $\mathrm{DE}$ appearing in the decay around $3 \mathrm{~s}$ appears to be due to constructive interference, which produces the same effect seen in Fig. 4(c).

\section{Discussion AND CONCLUSION}

We presented and discussed interference within sparse atomic estimations, which is a direct result of decomposing a signal using terms drawn from an overcomplete dictionary. In previous work, we sought to quantify the degree of interference in an estimation using DE. In this paper, we extended those results to create a short-term measure of DE to examine how interference is distributed throughout an estimation with respect to the signal itself. The phenomenon of DE and our measure were illustrated for three simple signals and a more complex music signal.

Using these measures, we can begin to answer such questions as: What part and percentage of a sparse atomic estimation is used to correct itself? Which terms are physically relevant to the signal, and which terms are products of the decomposition algorithm? Which parts of the signal "make sense" to decompose with the given dictionary? These questions are directly relevant to increasing the sparsity and efficiency of an estimation. STDE provides a measure with which to judge the fit of atoms to particular structures in the signal being estimated, as opposed to using only the inner product in (2). Furthermore, given a completed decomposition, we can review the interference in the estimation and return to the residual those terms associated with high interference. Such terms, it can be argued, do not "make sense" with that portion of the signal they have been chosen to model. We are currently investigating these extensions and applications.

\section{ACKNOWLEDGMENT}

This work was supported in part by the National Science Foundation under Grant CCF-0729229.

\section{REFERENCES}

[1] S. S. Chen, D. L. Donoho, and M. A. Saunders, "Atomic decomposition by basis pursuit," SLAM J. Scientific Comput., vol. 20, no. 1, pp. 33-61, Aug. 1998.

[2] S. Mallat and Z. Zhang, "Matching pursuits with time-frequency dictionaries," IEEE Trans. Signal Process., vol. 41, no. 12, pp. 33973415, Dec. 1993.

[3] L. Daudet and B. Torrésani, "Hybrid representations for audiophonic signal encoding," Signal Process., vol. 82, no. 11, pp. 1595-1617, Nov. 2002.

[4] R. Gribonval, "Sparse decomposition of stereo signals with matching pursuit and application to blind separation of more than two sources from a stereo mixture," in Proc. IEEE Int. Conf. Acoust., Speech, Signal Process., Orlando, FL, May 2002, vol. 3, pp. 3057-3060.

[5] B. L. Sturm, C. Roads, and G. Kling, "Sound visualization and transformation by means of matching pursuit," J. Audio Eng. Soc., 2007, submitted.

[6] R. Gribonval, E. Bacry, S. Mallat, P. Depalle, and X. Rodet, "Analysis of sound signals with high resolution matching pursuit," in Proc. IEEE-SP Int. Symp. Time-Frequency and Time-Scale Analysis, Paris, France, June 1996, pp. $125-128$.

[7] S. Jaggi, W. C. Carl, S. Mallat, and A. S. Willsky, "High resolution pursuit for feature extraction," Applied and Computational Harmonic Analysis, vol. 5, no. 4, pp. 428-449, Oct. 1998.

[8] M. M. Goodwin and M. Vetterli, "Matching pursuit and atomic signal models based on recursive filter banks," IEEE Trans. Signal Process., vol. 47 , no. 7, pp. 1890-1902, July 1999.

[9] B. L. Sturm, J. J. Shynk, L. Daudet, and C. Roads, "Dark energy in sparse atomic decompositions," IEEE Trans. Audio, Speech, Lang. Process., 2007, accepted for publication. 